\title{
Goodness-of-fit testing strategies from indirect observations
}

\author{
Jean-Michel Loubes* $^{*}$ and Clément Marteau ${ }^{\dagger}$
}

\begin{abstract}
We consider in this paper a goodness-of-fit testing problem in a density framework. In particular, we deal with an error-in-variables model where each new incoming observation is gathered with a random independent error. It is well-known that in such a situation, we are faced with an inverse (deconvolution) problem. Nevertheless, following recent results in the Gaussian white noise model, we prove that using procedures containing a deconvolution step is not always necessary.
\end{abstract}

\section{Introduction}

In this paper, we consider the goodness-of-fit testing problem of an unknown density $f$ from i.i.d random variables $X_{1}, \ldots, X_{n}$ observed with measurement errors. Actually let $\mathbf{Y}=\left(Y_{1}, \ldots, Y_{n}\right)$ be a sample of $n$ i.i.d. observations where

$$
Y_{i}=X_{i}+\epsilon_{i}, \forall i \in\{1 \ldots n\}
$$

The $\epsilon_{i}$ 's play the role of measurement errors, which are supposed to be centered and associated to a known density $g$. In this context, the density of the $Y_{i}$ corresponds to the convolution product between $f$ and $g$, denoted by $f * g$.

Inference on the density $f$ is a deconvolution problem and has been widely studied in the statistical literature. Estimation issues have received a great amount of attention. We mention for instance [Fan 1991] for a first study of the optimal rates of convergence related to deconvolution kernel estimators of $f$ or Delaigle et al. 2004 for a practical calibration of such estimators. More generally, we mention [Meister 2009] for an introduction to nonparametric deconvolution problems or Engl et al. 1996 and Cavalier 2008 where precise descriptions on inverse problems and related statistical issues are provided.

\footnotetext{
*Institut de Mathématiques de Toulouse, loubes@math.univ-toulouse.fr

†Institut de Mathématiques de Toulouse, marteau@math.univ-toulouse.fr
} 
Our aim in this paper is not to provide an estimator of the density $f$ but rather to assess optimality of procedures able to decide whether the unknown density $f$ is equal to $f_{0}$ where $f_{0}$ denotes some given density. For instance, the $X_{i}$ may correspond to the measures of a quantity of interest (e.g. weight, cholesterol rate,...) of $n$ individuals. The purpose is then to compare the distribution of this sample to the one of a benchmark population (e.g. diabetic or healthy people). From a statistical point of view, providing an answer to this question corresponds to test

$$
H_{0}^{I P}: f=f_{0}, \text { against } H_{1}^{I P}: f \neq f_{0}, f \in \mathcal{H}
$$

where $\mathcal{H}$ denotes a subset of $L^{2}(\mathbb{R})$. The exponent $I P$ traduces the fact that we deal with a contaminated sample with density $f * g$. Hence, inference on $f$ leads to the inverse problem of testing a density in a deconvolution model. This issue has been widely tackled in the statistical literature. We refer for instance to [Bissantz et al. 2009] or Holzmann et al. 2007] in a slightly different setting. Moreover, the papers [Butucea 2007, Butucea et al. 2007, Butucea et al. 2009 provide a complete study of this testing problem in many situations, including minimax rates of testing. However, as for any inverse problem, such procedures require to invert the convolution operator and are thus based on a regularization scheme of the observations (see Engl et al. 1996 or [Bissantz et al. 2007] for more details). From a practical point of view, such methods are sometimes difficult to implement since one needs to compute a deconvolution kernel in a density framework or the singular value decomposition for more general operators. It is thus natural to ask whether this regularization step is necessary.

Using reasonable assumptions (which will be made precise later on) on the density $g$, the convolution operator is injective. Hence, in some sense, the assertions " $f=f_{0}$ " and $" g * f=g * f_{0} "$ are equivalent. In a goodness-of-fit framework, two different points of view may be alternatively considered. The statistician may either perform an indirect test

$$
H_{0}^{I P}: f=f_{0}, \text { against } H_{1}^{I P}: f \neq f_{0}, f \in \mathcal{H}
$$

or may use a direct approach directly on the data, without a deconvolution step. In that case he considers the direct test

$$
H_{0}^{D P}: g * f=g * f_{0}, \text { against } H_{1}^{D P}: g * f \neq g * f_{0}, g * f \in \mathcal{H}^{\prime},
$$

for some $\mathcal{H}^{\prime} \subset L^{2}(\mathbb{R})$.

As proved in Laurent et al. 2011] in a Gaussian white noise setting, a surprising outcome is that the inversion is not always necessary in a goodness-of-fit purpose. Indeed, well calibrated direct procedures (i.e. dealing with the observation without regularization step) can do as well as classical tests based on an inversion of the operator. The aim of this paper is to extend this discussion to the deconvolution framework and to investigate the favorable cases. Actually, depending on the difficulty of the inverse problem defined by 
the regularity of the density of the noise $g$, and on the set of assumptions on the density $f$ to be detected, we prove that the specific treatment devoted to an inverse problem which includes an underlying inversion of the operator, may worsen the detection strategy.

This paper is organized as follows. In Section 2, we formalize the testing problem and present the existing results in the literature. In Section 3 we prove that direct testing procedures are often minimax for the inverse testing problem and we construct a test minimax for $H_{0}^{I P}$ that will fail on $H_{0}^{D P}$ in some particular situation. Most of the proofs are gathered in Section 5 .

\section{Two possible settings}

Recall that we consider the deconvolution model

$$
Y_{i}=X_{i}+\epsilon_{i}, \quad i=1, \ldots, n
$$

where the $X_{i}$ 's and $\epsilon_{i}$ 's are i.i.d real random variables with respective density functions (with respect to Lebesgue measure) $f$ and $g$. Since the $X_{i}$ 's and $\epsilon_{i}$ 's are independent, the $Y_{i}$ 's are associated to the density $f * g$ where $*$ denotes the convolution operator. Our aim is to test $f=f_{0}$ or equivalently $f * g=f_{0} * g$ where $f_{0}$ denotes a benchmark density function.

We consider the following general smoothness assumption on the densities given by the decay of their Fourier transform. We assume that both $f_{0}$ and $f$ belong to the space $\mathcal{E}(h, L)$ defined as

$$
\mathcal{E}(h, L)=\left\{f: \int_{\mathbb{R}}\left|\Phi_{f}(t)\right|^{2}|h(t)|^{2} d t<2 \pi L\right\},
$$

where $\Phi_{f}$ denotes the Fourier transform of a function $f, L$ a positive constant and $h$ an increasing function. Remark that in the particular case where $h(t)=t^{s}$ for some $s>0$, $\mathcal{E}(h, L)$ corresponds to the Sobolev space

$$
W(s, L)=\left\{f \in \mathcal{C}^{s}, \int\left|\Phi_{f}(t)\right|^{2}|t|^{2 s} d t<2 \pi L\right\}
$$

while, when $h(t)=\exp \left(\alpha|t|^{r}\right)$ for all $t \in \mathbb{R}$, the set $\mathcal{E}(h, L)$ is identified as the space of super-smooth densities functions

$$
S(\alpha, r, L)=\left\{f \in \mathcal{C}^{\infty}, \int\left|\Phi_{f}(t)\right|^{2} \exp \left(2 \alpha|t|^{r}\right) d t<2 \pi L\right\} .
$$

The development of testing strategies is strongly associated to the notion of separation rates corresponding to the minimax point of view developed in the series of papers due 
to Ingster 1993 . Given the hypothesis $H_{0}$, let $\rho_{n}$ be a decreasing sequence, and $H_{1}$ an alternative of the form

$$
H_{1}: f \neq f_{0}, f \in \mathcal{H}\left(h, L, \rho_{n}\right)
$$

where

$$
\mathcal{H}\left(h, L, \rho_{n}\right)=\left\{\nu \in \mathcal{E}(h, L),\left\|\nu-f_{0}\right\|>\rho_{n}\right\},
$$

and $\|$.$\| denotes the L_{2}$-norm. For a testing procedure $\Delta_{n}$, we write by convention $\Delta_{n}=0$ if we accept $H_{0}$ and $\Delta_{n}=1$ if we reject the assumption. For a given $0<\xi<1$, a testing procedure $\Delta_{n}^{\star}$ is said to attain the testing rate $\rho_{n}$ over $\mathcal{E}(h, L)$ if there exists $C^{\star}=C^{\star}(\xi)$ such that

$$
\limsup _{n \rightarrow+\infty}\left[P_{f_{0}}\left(\Delta_{n}^{\star}=1\right)+\sup _{f \in \mathcal{H}\left(h, L, C \rho_{n}\right)} P_{f}\left(\Delta_{n}^{\star}=0\right)\right] \leq \xi,
$$

for all $C>C^{\star}$. In other words, the procedure $\Delta_{n}$ is able to detect that $f \neq f_{0}$ with prescribed asymptotic errors as soon as $\left\|f-f_{0}\right\|>C \rho_{n}$. The rate $\rho_{n}$ is then said to be the minimax rate of testing on $\mathcal{E}(h, L)$ if there exists $C_{\star}=C_{\star}(\xi)>0$ such that

$$
\liminf _{n \rightarrow+\infty} \inf _{\Delta_{n}}\left[P_{f_{0}}\left(\Delta_{n}=1\right)+\sup _{\mathcal{H}\left(h, L, C \rho_{n}\right)} P_{f}\left(\Delta_{n}=0\right)\right] \geq \xi,
$$

for all $0<C<C_{\star}$. The infimum in the previous formula is taken over all testing procedures $\Delta_{n}$. The quality of the testing procedure then relies on the quantity $\rho_{n}$, and of course, for a given level and power of test, the smaller the $\rho_{n}$, the better.

In the noise-free case (i.e. $\epsilon_{i}=0$ for all $i=1 \ldots n$ ), minimax rates of testing are described for instance in Ingster 1993. Similar results in the Gaussian sequence space model are obtained in Baraud 2002] or [Baraud et al. 2003 where adaptation with respect to the smoothness is discussed. In the inverse problem framework, two different cases are commonly discussed, which are associated to the rate of decay of the Fourier transform of $g$. One may alternatively assume that

$$
0<\left|\Phi_{g}(t)\right|=\mathcal{O}\left(|t|^{-\beta}\right), \text { as } t \rightarrow+\infty
$$

which yields a mildly ill-posed inverse problem or that

$$
0<\left|\Phi_{g}(t)\right|=\mathcal{O}\left(\exp \left(-\gamma|t|^{\beta}\right)\right), \text { as } t \rightarrow+\infty,
$$

which corresponds to a severely ill-posed inverse problem. Note that imposing that the Fourier transform of the noise vanishes nowhere induces that the convolution operator is injective. This warrants the identifiability of the statistical model. In the specific error-in-variables model (44), which is at the heart of the present paper, the most complete reference is up to our knowledge [Butucea 2007]. For the sake of convenience, we reproduce the obtained result in Table 1. We mention that similar rates are available 


\begin{tabular}{ccc}
\hline $\mathrm{f} / \mathrm{g}$ & $\left|\Phi_{g}(u)\right| \sim|u|^{-\beta}$ & $\left|\Phi_{g}(u)\right| \sim \exp ^{-\gamma|u|^{\beta}}$ \\
\hline$W(s, L)$ & $n^{-\frac{2 s}{4 s+4 \beta+1}}$ & $(\log n)^{-s / \beta}$ \\
\hline$S(\alpha, r, L)$ & $\log (n)^{(\beta+1 / 4) / r} n^{-1 / 2}$ & $\exp \left(-\alpha / v_{*}^{r}\right)(r<\beta)$ \\
& & $n^{-1 / 2} v_{*}^{(\beta-1)_{-}} \exp \left(-\gamma / h_{*}^{\beta}\right)(r>\beta)$ \\
\hline
\end{tabular}

Table 1: Minimax rate of testing in the error-in-variables model (4). The term $v_{*}$ denotes the solution of $2 \alpha v_{*}^{-r}+2 \gamma v_{*}^{-\beta}=\log (n)-\log \log (n)$.

in a Gaussian white noise model: see [Laurent et al. 2012] or Ingster et al. 2012.

Here, goodness-of-fit tests in a deconvolution model can be built using the two different settings which amount to test either " $f=f_{0}$ ", which will be referred to as the inverse problem setting, or " $g * f=g * f_{0}$ " which will be named the direct problem setting. This problematic has been discussed for the first time in [Holzmann et al. 2007]. Namely, a direct procedure will correspond to a test based on the blurred observations and designed for the detection of differences between $f * g$ and $f_{0} * g$ while an inverse problem procedure deals with a test based on an estimator of $f$. More precisely for $h$ and $\tilde{h}$ two functions that will control the respective regularities of $f$ and $f * g$, we define the following set of hypotheses

$$
H_{0}^{D P}: g * f=g * f_{0}, \text { against } H_{1}^{D P}: g * f \neq g * f_{0}, g * f \in \mathcal{H}_{1}\left(\tilde{h}, L, \rho_{n}^{D P}\right),
$$

while the second one can be considered as an inverse problem, where we test

$$
H_{0}^{I P}: f=f_{0} \text {, against } H_{1}^{I P}: f \neq f_{0}, f \in \mathcal{H}_{1}\left(h, L, \rho_{n}^{I P}\right) .
$$

In this paper, we prove that although the convolution operator is one-to-one, the two testing problems are not equivalent. In particular, we will see that a testing procedure minimax for (6) is also minimax for the problem (7) with a particular choice for $\tilde{h}$. We also investigate cases where the converse is not true: we will exhibit optimal deconvolution testing procedures that are not minimax in the direct case.

\section{Comparison of the inverse and direct approaches}

The aim of this section is to compare the testing problems (66) and (7). Our aim is to describe the cases where direct procedures may be minimax for (77) and inverse tests are minimax for (6)).

Throughout this section, we will consider a noise having a non null Fourier transform, i.e.

$$
\Phi_{g}(t) \neq 0, \forall t \in \mathbb{R}
$$


This assumption is quite common in the statistical literature, in particular when considering nonparametric deconvolution problems. Actually it allows for instance the construction of a deconvolution kernel, which gives rise to a natural estimator by simply removing the part of the signal due to the noise by a mere division.

More specifically, we deal with the following hypothesis.

Assumption A1: There exists a positive constant $C_{l}$ such that for all $t \in \mathbb{R}$

$$
\left|\Phi_{g}(t)\right| \geq C_{l}|t|^{-\beta}
$$

for mildly ill-posed problems or

$$
\left|\Phi_{g}(t)\right| \geq C_{l} \exp \left(-\gamma|t|^{\beta}\right)
$$

for severly ill-posed inverse problems.

\subsection{Performances of direct methods}

In this part, we prove that for a well-chosen 'regularity' $\tilde{h}$, (direct) testing procedures that are minimax for (6) are also minimax for (7).

In order to provide a satisfying comparison of the problems (6) and (7), one needs first to have a precise idea of the regularity of $f * g$ provided $f \in \mathcal{E}(v, L)$ for some $h$. Since $\Phi_{f * g}=\Phi_{f} \cdot \Phi_{g}$,

$$
f \in \mathcal{E}(h, L) \Leftrightarrow \int\left|\Phi_{f * g}(t)\right|^{2}\left|\Phi_{g}(t)\right|^{-2}|h(t)|^{2} d t<L \Leftrightarrow f * g \in \mathcal{E}\left(\Phi_{g}^{-1} h, L\right) .
$$

In particular, if $|h(t)|=|t|^{s}$, i.e. $\mathcal{E}(h, L)=W(s, L)$, we get that

$$
f \in W(s, L) \Leftrightarrow f * g \in W\left(s+\beta, L^{\prime}\right)
$$

for some $L^{\prime}$ provided $\left|\Phi_{g}(t)\right|=\mathcal{O}\left(|t|^{-\beta}\right)$ and $\left|\Phi_{g}(t)\right| \neq 0$ for all $t \in \mathbb{R}$.

Since the assertions $g * f=g * f_{0}$ and $f=f_{0}$ are equivalent provided Assumption A1 is satisfied, it seems clear that both hypotheses $H_{0}^{D P}$ and $H_{0}^{I P}$ are equivalent. Consequently, the first kind errors are the same for a given test $\Delta_{n}$ whatever the chosen null hypothesis. The alternatives are therefore at the heart of the comparison between the two testing problems.

The following theorem establishes that in the cases considered in this paper, a direct test performs as well as specific procedures tailored to handle the particular deconvolution issue. 
Theorem 3.1 Let $\xi \in] 0,1[$ be a fixed level of test. We assume that $h$ is polynomially (Sobolev) or exponentially increasing (supersmooth). The related inverse problem is mildly or severely ill-posed and we assume that Assumption $\boldsymbol{A} 1$ is satisfied. Then every test minimax for $H_{0}^{D P}$ on $\mathcal{E}\left(\left|\Phi_{g}(.)\right|^{-1} h, L\right)$ is also minimax for $H_{0}^{I P}$ on $\mathcal{E}(h, L)$.

The proof of this theorem relies on the following lemma which gives a control on $\|\nu * g\|$ from $\|\nu\|$ provided the function of interest $\nu$ belongs to $\mathcal{E}(h, L)$ for some $h$ and $L$.

Lemma 3.2 Let $\gamma_{n}$ be a positive sequence such that $\gamma_{n} \rightarrow 0$ as $n \rightarrow+\infty$. The following embedding holds:

$$
\left\{\nu \in \mathcal{E}(h, L),\|\nu\|^{2} \geq \gamma_{n}\right\} \subset\left\{\nu \in \mathcal{E}(h, L),\|\nu * g\|^{2} \geq \mu_{n}\right\},
$$

where

$$
\mu_{n}=\frac{1}{2}\left|\Phi_{g}\left(\tau_{n}\right)\right|^{2} \gamma_{n},
$$

and $\tau_{n}$ is such that $L\left|h\left(\tau_{n}\right)\right|^{-2} \leq \gamma_{n} / 2$.

Proof Let $t \in \mathbb{R}^{+}$which will be chosen later and $\nu \in \mathcal{E}(h, L)$ such that $\|\nu\|^{2} \geq \gamma_{n}$. For the sake of convenience, we will also suppose that the function $t \mapsto\left|\Phi_{g}(t)\right|$ is non increasing. Then

$$
\begin{aligned}
\|\nu * g\|^{2} & =\int\left|\Phi_{\nu}(u)\right|^{2}\left|\Phi_{g}(u)\right|^{2} d u \\
& \geq \int_{|u| \leq t}\left|\Phi_{\nu}(u)\right|^{2}\left|\Phi_{g}(u)\right|^{2} d u, \\
& \geq\left|\Phi_{g}(t)\right|^{2} \int_{|u| \leq t}\left|\Phi_{\nu}(u)\right|^{2} d u, \\
& \geq\left|\Phi_{g}(t)\right|^{2}\left(\|\nu\|^{2}-\int_{|u|>t}\left|\Phi_{\nu}(u)\right|^{2} d u\right) .
\end{aligned}
$$

Note that the assumption about the decay of $\Phi_{g}$ could be relaxed, up to some more complicated algebra in the proofs, replacing $\left|\Phi_{g}(t)\right|^{2}$ by $\inf _{|u| \leq t}\left|\Phi_{\nu}(u)\right|^{2}$ in the following. Since $\nu \in \mathcal{E}(h, L)$

$$
\int_{|u|>t}\left|\Phi_{\nu}(u)\right|^{2} d u \leq|h(t)|^{-2} \int_{|u|>t}|h(u)|^{2}\left|\Phi_{\nu}(u)\right|^{2} d u \leq L|h(t)|^{-2} .
$$

Hence

$$
\|\nu * g\|^{2} \geq\left|\Phi_{g}(t)\right|^{2}\left(\gamma_{n}-L|h(t)|^{-2}\right) .
$$

We conclude the proof choosing $t=\tau_{n}$ such that $L\left|h\left(\tau_{n}\right)\right|^{-2} \leq \gamma_{n} / 2$. 
Applying Lemma 3.2, we get that for all testing procedures $\Delta_{n}$,

$$
\sup _{f \in \mathcal{E}(h, L),\left\|f-f_{0}\right\|^{2} \geq \gamma_{n}} P_{f}\left(\Delta_{n}=0\right) \leq \sup _{f \in \mathcal{E}(h, L),\left\|\left(f-f_{0}\right) * g\right\|^{2} \geq \mu_{n}} P_{f}\left(\Delta_{n}=0\right) .
$$

Consequently, provided $\gamma_{n}$ is of order $\left(\rho_{n}^{I P}\right)^{2}$ and $\mu_{n}$ is of order $\left(\rho_{n}^{D P}\right)^{2}$, a test minimax for $H_{0}^{D P}$ will be also minimax for $H_{0}^{I P}$. The whole proof of Theorem 3.1 is postponed to the Appendix.

The consequence of the previous result is that classical testing procedures designed for the direct problem (6) can be directly used on our noisy observations without performing any regularization step. No need for a special treatment is required since direct testing procedures are proved to be minimax. Hence, the following direct testing procedure allows, following the results presented in Theorem 3.1, to tackle the testing issue of a wide variety of inverse problems.

Let $K$ denote a kernel i.e. a function $K: \mathbb{R} \rightarrow \mathbb{R}$ such that

$$
\int_{\mathbb{R}} K(x) d x=1 \text { and } \int_{\mathbb{R}} K^{2}(x) d x<\infty .
$$

Define the test statistics as

$$
\tilde{T}_{n, \lambda}=\frac{1}{n(n-1)} \sum_{k \neq j}\left\langle K_{\lambda}\left(.-Y_{k}\right)-f_{0}, K_{\lambda}\left(.-Y_{j}\right)-f_{0}\right\rangle,
$$

where $K_{\lambda}:=\lambda^{-1} K_{g}(. / \lambda)$ for some bandwidth $\lambda$. Remark that the term $\tilde{T}_{n, \lambda}$ is an estimator of $\left\|\left(f-f_{0}\right) * g\right\|^{2}$. Then, we set

$$
\tilde{\Delta}_{n, \lambda}=\mathbf{1}_{\left\{\left|\tilde{T}_{n, \lambda}\right|>\tilde{C} u_{n}^{2}\right\}}
$$

where $\tilde{C}$ denotes a sufficiently large constant and $\left(u_{n}\right)_{n \in \mathbb{N}}$ a positive real sequence. This decision rule means that we reject $H_{0}^{D P}$ if the estimator of $\left\|\left(f-f_{0}\right) * g\right\|^{2}$ takes large values. Otherwise, if $\tilde{T}_{n, \lambda}$ is small w.r.t. the sequence $\left(u_{n}\right)_{n \in \mathbb{N}}$, we accept $H_{0}^{D P}$. This kind of estimator has been widely studied in the literature and appropriate values for $\lambda$ and $u_{n}$ have been proposed in several different setting. In particular, it has been proved (we refer to [ngster 1993 or [Butucea 2007] among others) that the test $\tilde{\Delta}_{n, \lambda}$ is minimax for $H_{0}^{D P}$ on both Sobolev and super-smooth function classes.

\subsection{Limitation of inverse approaches}

In this section, we prove that the direct and inverse testing problems for goodnessof-fit in a deconvolution model are not equivalent in the sense that a testing procedure optimal for the inverse problem (77) may fail when dealing with the direct observation 
model (6). This property is essentially due to the fact that a testing procedure designed for an inverse problem (i.e. containing a regularization step) is calibrated in order to take into account a possible large variance for the errors. But the drawback is that such a procedure may be too conservative or unable to detect alternatives with small intensity.

The key of the proof is to exhibit a testing procedure minimax in the inverse setting (7) that may fail in certain situations for the direct testing issue. We deal in this paper with a procedure proposed by Butucea 2007] in the error-in-variable model. In a first time, we need to construct a preliminary estimator of $f$ using a kernel type estimator.

First, consider $K$ a kernel. The associated deconvolution kernel $K_{g}$ is defined as

$$
\Phi_{K_{g}}=\frac{\Phi_{K}(t)}{\Phi_{g}(t / \lambda)}
$$

for some bandwidth $\lambda$. We refer to [Meister 2009] for more details on the construction of such quantities and discussion on the Assumption A1. The testing procedure is then based on an estimation of $\left\|f-f_{0}\right\|^{2}$. A candidate is given by

$$
T_{n}^{\star}=\frac{1}{n(n-1)} \sum_{k \neq j}\left\langle K_{g, \lambda}\left(.-Y_{k}\right)-f_{0}, K_{g, \lambda}\left(.-Y_{j}\right)-f_{0}\right\rangle,
$$

where $K_{g, \lambda}:=\lambda^{-1} K_{g}(. / \lambda)$. Then, we set

$$
\Delta_{n}^{\star}=\mathbf{1}_{\left\{\left|T_{n}^{\star}\right|>C^{\star} t_{n}^{2}\right\}},
$$

where $C^{\star}$ and $t_{n}$ are positive parameters that should be properly chosen. For an appropriate choice of $t_{n}$ and $h_{n}$ (see e.g. [Butucea 2007]) this test is known to be minimax for $H_{0}^{I P}$ on the different smoothness classes considered in this paper. In particular,

$$
\lim _{n \rightarrow+\infty}\left[P_{f_{0}}\left(\Delta_{n}^{\star}=1\right)+\sup _{f \in \mathcal{H}\left(h, L, C \rho_{n}\right)} P_{f}\left(\Delta_{n}^{\star}=0\right)\right] \leq \xi .
$$

Nevertheless, we prove that this procedure fails in the direct case, i.e. that there exists $f_{1, n} \in \mathcal{E}\left(\Phi_{g}(.)^{-1} h, L\right)$ such that

$$
\left\|\left(f_{1, n}-f_{0}\right) * g\right\| \geq \mathcal{C}_{1} \rho_{n}\left(\mathcal{E}\left(\Phi_{g}(.)^{-1} h, L\right)\right) \text { but } \lim _{n \rightarrow+\infty}\left[P_{f_{0}}\left(\Delta_{n}^{\star}=1\right)+P_{f_{1, n}}\left(\Delta_{n}^{\star}=0\right)\right]>\xi,
$$

whatever the value of $\mathcal{C}_{1}$.

Theorem 3.3 Let $0<\xi<1$ be a test level and a function $h$ to be fixed. Assume that Assumption A1 holds. For both mildly and severely ill-posed problems, there exist level$\alpha$ tests minimax for $H_{0}^{I P}$ on $\mathcal{E}(h, L)$ but not for $H_{0}^{D P}$ on $\mathcal{E}\left(h . \Phi_{g}^{-1}, L^{\prime}\right)$.

Clearly, both testing problems (6) and (7) are not equivalent. In some sense, direct methods are more robust with respect to measurement errors. Inverse methods are too conservative in the direct case since they are designed for high variance testing problems. 


\begin{tabular}{cc}
\hline$f * g \in W\left(s^{\prime}, L\right)$ & $n^{-\frac{2 s^{\prime}}{4 s^{\prime}+1}}$ \\
\hline$f * g \in S\left(\alpha^{\prime}, r, L\right)$ & $\log (n)^{1 / 4 r} n^{-1 / 2}$ \\
\hline
\end{tabular}

Table 2: Minimax rate of testing for the problem (6).

\subsection{Conclusion}

The main contribution of this paper is that the regularization is not necessary in the minimax sense when considering goodness-of-fit testing problems. This allows to propose direct procedures that are not based on a deconvolution scheme, i.e. that require the inversion of the underlying operator.

This point of view may allow to consider specific problems for which this deconvolution might be difficult or even impossible. In this sense, the case of uniform measurement errors is of first interest. Indeed, in this specific case, $\Phi_{g}(t)=0$ for some $t \in \mathbb{R}$ which does not allow the construction of (10). Nevertheless, Lemma 3.2 can not be directly generalized in this situation or at least up to some additional constraint on the signal of interest (for instance source conditions, see [Engl et al. 1996 or [Loubes et al. 2009] for instance). Yet a lower bound would be necessary for the testing problem in such a case, which still remains an open problem.

\section{Proof of the main results}

In this section, the quantities $C$ and $c$ will denote generic constants that may vary from line to line, and even in the same line. Given two real sequences $\left(a_{n}\right)_{n \in \mathbb{N}}$ and $\left(b_{n}\right)_{n \in \mathbb{N}}$, we write $a_{n} \sim b_{n}$ if there exists $c, C$ positive constants such that $c \leq a_{n} / b_{n} \leq C$ for all $n \in \mathbb{N}$.

\subsection{Proof of Theorem 3.1}

For the sake of convenience, we recall in Table2 the different testing rates in the direct case, according to the regularity of the considered densities.

Let $\xi \in] 0,1\left[\right.$ be fixed and $\Delta_{n}$ a testing procedure minimax for $H_{0}^{D P}$ on $\mathcal{E}\left(\left|\Phi_{g}^{-1}\right| h, L\right)$. Then, according to (5)

$$
\lim _{n \rightarrow+\infty}\left[P_{f_{0}}\left(\Delta_{n}=1\right)+\sup _{f * g \in \mathcal{H}\left(\left|\Phi_{g}\right|^{-1} h, L, C \rho_{n}^{D P}\right)} P_{f}\left(\Delta_{n}=0\right)\right] \leq \xi,
$$


for some positive constant $C$. The proof is then based on the following scheme. We first remark that $f \in \mathcal{E}(h, L) \Rightarrow g * f \in \mathcal{E}\left(\left|\Phi_{g}\right|^{-1} h, L^{\prime}\right)$ for some $L^{\prime}$. Hence, using Lemma 3.2, we get

$$
\begin{aligned}
\left\{f \in \mathcal{E}(h, L),\left\|f-f_{0}\right\|^{2} \geq\right. & \left.C_{2}\left(\rho_{n}^{I P}\right)^{2}\right\} \\
& \subset\left\{g * f \in \mathcal{E}\left(\left|\Phi_{g}\right|^{-1} h, L^{\prime}\right),\left\|\left(f-f_{0}\right) * g\right\|^{2} \geq C_{2}\left(\left|\Phi_{g}\left(\tau_{n}\right)\right| \rho_{n}^{I P}\right)^{2}\right\} .
\end{aligned}
$$

Consequently, the test $\Delta_{n}$ will be minimax as soon as

$$
\left|\Phi_{g}\left(\tau_{n}\right)\right| \rho_{n}^{I P}>C \rho_{n}^{D P}, \text { where } L\left|h\left(\tau_{n}\right)\right|^{-2} \leq 1 / 2\left(\rho_{n}^{I P}\right)^{2},
$$

and $C$ denotes a positive constant independent of $n$. We consider 4 different cases according to the possible regularities of the unknown density $f_{0}$ (Sobolev or super-smooth) and the degree of ill-posedness of the problem (mildly or severely ill-posed ). For each considered case, we prove that the bound (12) holds. For the sake of convenience, the minimax rates of convergence in the direct and inverse setting are written respectively $\rho_{n}^{D P}$ and $\rho_{n}^{I P}$. If we do not write explicitly the dependency with respect to the functional spaces, it will yet be recalled for each different setting.

1st case: The problem is mildly ill-posed i.e. $\left|\Phi_{g}(t)\right|=\mathcal{O}\left(|t|^{-\beta}\right)$ for some $\beta>0$ and $f \in W(s, L)$ for some $s>0$, i.e. $|h(t)|=t^{s}$. In this case, $f * g \in \mathcal{E}(\tilde{h}, L) \subset W\left(s+\beta, L^{\prime}\right)$ for some $L^{\prime}$. The term $\tau_{n}$ is chosen as

$$
L\left|\tau_{n}\right|^{-2 s}=1 / 2 \gamma_{n} \Leftrightarrow \tau_{n} \sim\left[\rho_{n}^{I P}\right]^{-1 / s} \sim n^{\frac{2}{4 s+4 \beta+1}} .
$$

Then

$$
\left|\Phi_{g}\left(\tau_{n}\right)\right|^{2}\left(\rho_{n}^{I P}\right)^{2} \sim \tau_{n}^{-2 \beta} n^{-\frac{4 s}{4 s+4 \beta+1}} \sim n^{-\frac{4 s+4 \beta}{4 s+4 \beta+1}} \sim\left(\rho_{n}^{D P}\right)^{2}
$$

2nd case: The problem is mildly ill-posed and $f \in S(\alpha, r, L)$. In this setting, remark that the function $f * g \in S\left(\alpha, r, L^{\prime}\right)$ for some $L^{\prime}$. Then, according to the rates obtained in [Butucea 2007,

$$
\left(\rho_{n}^{I P}\right)^{2} \sim \frac{\log (n)^{(2 \beta+1 / 2) / r}}{n}, \text { and }\left(\rho_{n}^{D P}\right)^{2} \sim \frac{\log (n)^{1 / 2 r}}{n} .
$$

We choose $\tau_{n}$ as

$$
\tau_{n}=\left\lceil\left(\frac{1}{2 \alpha} \ln (n)\right)^{1 / r}\right\rceil,
$$

where for any $x \in \mathbb{R},\lceil x\rceil$ denotes the smaller integer greater than $x$. Then for $n$ large enough,

$$
L^{2}\left|h\left(\tau_{n}\right)\right|^{-2} \leq L^{2} n^{-1} \leq L^{2} n^{-1} \log (n)^{(2 \beta+1 / 2) / r} \leq C\left(\rho_{n}^{I P}\right)^{2},
$$


for some $C>0$. Moreover

$$
\left|\Phi_{g}\left(\tau_{n}\right)\right|^{2}\left(\rho_{n}^{I P}\right)^{2} \sim \log (n)^{1 / 2 r} \frac{1}{n} \sim\left(\rho_{n}^{D P}\right)^{2} .
$$

3rd case: We assume that $f \in W(s, L)$ for some $s>0$ and that the problem is severely ill-posed: $\left|\Phi_{g}(t)\right|=\mathcal{O}\left(e^{-\gamma|t|^{\beta}}\right)$ as $t \rightarrow+\infty$, for some $\gamma>0$. In this setting, remark that $c e^{\gamma|t|^{\beta}} \leq c(t):=h(t) \Phi_{g}^{-1}(t) \leq C e^{2 \gamma|t|^{\beta}}$ for all $t$. Hence, using the results of [Butucea 2007], we get

We set

$$
\rho_{n}^{I P} \sim(\log (n))^{-s / \beta} \text { and } \rho_{n}^{D P} \sim \frac{1}{\sqrt{n}}(\log (n))^{1 / 4 \beta} .
$$

$$
\tau_{n}=\left\lfloor\frac{1}{2 \gamma} \log \left(n(\log n)^{-1 / 2 \beta}\right)+\frac{1}{2 \gamma} \log \left((\log n)^{-2 s / \beta}\right)\right\rfloor^{1 / \beta},
$$

where for any $x \in \mathbb{R},\lfloor x\rfloor$ denotes the greater integer smaller than $x$. Then

$$
L\left|h\left(\tau_{n}\right)\right|^{-2} \leq C L\left|\tau_{n}\right|^{-2 s} \leq C(\log (n))^{-2 s / \beta} \leq C\left(\rho_{n}^{I P}\right)^{2},
$$

where $C_{2}>0,0<c<1$, and

$$
\left|\Phi_{g}\left(\tau_{n}\right)\right|^{2}\left(\rho_{n}^{I P}\right)^{2} \geq C e^{-2 \gamma\left|\tau_{n}\right|^{\beta}}(\log n)^{-2 s / \beta} \geq C \frac{1}{n}(\log (n))^{1 / 2 \beta} \simeq\left(\rho_{n}^{D P}\right)^{2} .
$$

4th case: Assume that $f \in S(\alpha, r, L)$ for some $\alpha, r>0$ and that the problem is severely ill-posed. Consider in a first time the case where $\beta>r$. In this setting, remark that $\tilde{c_{1}} e^{\gamma|t|^{\beta}} \leq|h(t)|\left|\Phi_{g}(t)\right|^{-1} \leq \tilde{c_{2}} e^{2 \gamma|t|^{\beta}}$ for all $t \in \mathbb{R}$. Hence, from [Butucea 2007], we get

$$
\left(\rho_{n}^{I P}\right)^{2} \simeq e^{-4 \alpha / \tilde{t}^{\star}} \text { and }\left(\rho_{n}^{D P}\right)^{2} \simeq \frac{1}{n}(\log (n))^{1 / 2 \beta},
$$

where $t^{\star}$ denotes the integer part of the solution of $2 \alpha\left(t^{\star}\right)^{r}+2 \gamma\left(t^{\star}\right)^{\beta}=\log (n)$. Then, we set

$$
\tau_{n}=\left\lfloor\frac{1}{2 \gamma} \log \left(n(\log n)^{1 / 2 \beta}\right)-\frac{\alpha}{\gamma}\left(t^{\star}\right)^{r}\right\rfloor .
$$

Remark that for $\sigma$ small enough, $\tau_{n} \geq t^{\star}$ since $r<\beta$. Therefore

$$
\left|h\left(\tau_{n}\right)\right|^{-2}=R^{2} e^{-2 \alpha \tau_{n}^{r}} \leq c C_{2} e^{-2 \alpha\left(t^{\star}\right)^{r}},
$$

where $C_{2}>0$ and $0<c<1$. Then

$$
\left|\Phi_{g}\left(\tau_{n}\right)\right|^{2}\left(\rho_{n}^{I P}\right)^{2} \geq C e^{-2 \gamma \tau_{n}^{\beta}-2 \alpha t_{\star}^{s}} \geq C \frac{1}{n}(\log n)^{1 / 2^{\star} \beta} \sim\left(\rho_{n}^{D P}\right)^{2} .
$$

The proof of the case $r<\beta$ follows essentially the same lines. 


\subsection{Proof of Theorem 3.3}

As explained in Section 3.2 , we deal with the deconvolution testing procedure $\Delta_{n}^{\star}$ introduced in (11). We prove that this procedure fails in the direct case, i.e. that there exists $f_{1} \in \mathcal{E}\left(\Phi_{g}(.)^{-1} h, L\right)$ such that

$$
\left\|\left(f_{1}-f_{0}\right) * g\right\| \geq \mathcal{C}_{1} \rho_{n}\left(\mathcal{E}\left(\Phi_{g}(.)^{-1} h, L\right)\right) \text { but } \lim _{n \rightarrow+\infty}\left[P_{f_{0}}\left(\Delta_{n}^{\star}=1\right)+P_{f_{1}}\left(\Delta_{n}^{\star}=0\right)\right]>\xi,
$$

whatever the value of $\mathcal{C}_{1}$.

\subsubsection{Construction of a particular $f_{1}=f_{1, n}$}

To this end, we consider the function $f_{1, n}$ defined as

$$
f_{1, n}=f_{0}+r_{n} \omega
$$

where

$$
f_{0}(x) \geq \frac{C}{1+x^{2}}, \omega(x)=\cos (\sigma x) \cdot \frac{1-\cos (x)}{\pi x^{2}},
$$

$C, \sigma$ are positive constants such that $f_{1, n}(x) \geq 0$ for all $x \in \mathbb{R}$ and $r_{n}$ is a parameter which is allowed to depend on $n$. The term $r_{n} \omega$ corresponds to a perturbation of the density $f_{0}$. It is possible to see that

$$
\Phi_{\omega}(t)=(1-|t-\sigma|)_{+}, \forall t \in \mathbb{R} .
$$

In particular, remark that $\operatorname{supp}\left(\Phi_{\omega}\right) \subset[1-\sigma ; 1+\sigma] \subset[-2 ; 2]$ for $0<\sigma<1$. Hence, the perturbation $r_{n} \omega$ only concerns low frequencies. We can also verify that

$$
\Phi_{f_{1, n}}(0)=\Phi_{f_{0}}(0)+r_{n} \Phi_{\omega}(0)=1
$$

which ensures that $f_{1, n}$ is a density w.r.t. the Lebesgue measure. Moreover,

$$
\left\|g *\left(f_{1, n}-f_{0}\right)\right\|^{2}=r_{n}^{2} \int_{-2}^{2}\left|\Phi_{\omega}(t)\right|^{2}\left|\Phi_{g}(t)\right|^{2} \geq C r_{n}^{2} \inf _{t \in[-2 ; 2]}\left|\Phi_{g}(t)\right|^{2} .
$$

As in the proof of Theorem 3.1, we consider 4 different cases according to possible regularity of the function $f$ and the degree of ill-posedness of the problem. In each case, choosing $r_{n}=\mathcal{C}_{1} \rho_{n}^{D P}$, we get

$$
\left\|g *\left(f_{1, n}-f_{0}\right)\right\|>C \rho_{n}^{D P} \text { but }\left\|f-f_{0}\right\|=r_{n}^{2}\|\omega\|^{2} \leq C \rho_{n}^{D P}<<\rho_{n}^{I P}, \text { as } n \rightarrow+\infty,
$$

for some positive constant $C>0$. The inequality in the right hand side of the previous equation is often incompatible with the optimality of $\Delta_{n}^{\star}=\mathbf{1}_{\left\{\left|T_{n}^{\star}\right|^{2} \geq \mathcal{C}^{\star} t_{n}^{2}\right\}}$. 


\subsubsection{Lower bounds for the second kind error}

In the following, we prove that for all fixed $\xi$, with a good choice of $C^{\star}$, we have

$$
\left\|g *\left(f_{1, n}-f_{0}\right)\right\|>\mathcal{C}_{1} \rho_{n}^{D P} \text { but } \lim _{n \rightarrow+\infty}\left[P_{f_{0}}\left(\Delta_{n}^{\star}=1\right)+P_{f_{1, n}}\left(\Delta_{n}^{\star}=0\right)\right]>\xi,
$$

whatever the value of $\mathcal{C}_{1}$.

1st case: In a first time, we assume that the density of the noise is polynomially smooth, i.e. $\left|\Phi_{g}(t)\right|=\mathcal{O}\left(|t|^{-\beta}\right)$ as $t \rightarrow+\infty$ for some $\beta>0$. Provided

$$
t_{n}=n^{-\frac{2 s}{4 s+4 \beta+1}} \text { and } h=n^{-\frac{2}{4 s+4 \beta+1}},
$$

the test $\Delta_{n}^{\star}$ is minimax for $H_{0}^{I P}$ on $W(s, L)$ for $\mathcal{C}^{\star}$ large enough. Nevertheless, we prove that this test is not minimax for $H_{0}^{D P}$ on $W\left(s+\beta, L^{\prime}\right)$. To this end, we consider the function $f_{1, n}$ defined in (13) with

$$
r_{n}=\mathcal{C}_{1} n^{-\frac{2 s+2 \beta}{4 s+4 \beta+1}}
$$

for some positive constant $\mathcal{C}_{1}$. With such a construction, we have

$$
\left\|g * f_{1, n}-g * f_{0}\right\|^{2}>\mathcal{C}_{1} c_{g} n^{-\frac{4 s+4 \beta}{4 s+4 \beta+1}}, \text { and } \lim _{n \rightarrow+\infty} \inf P_{f_{1, n}}\left(\Delta_{n}^{\star}=0\right)>\xi
$$

for some constant $c_{g}$ depending on the density $g$. The left hand side of (15) is a direct consequence of (14). We only have to prove the right hand side. First, introduce

$$
V_{f_{1, n}}\left(T_{n}^{\star}\right)=\operatorname{Var}\left(T_{n}^{\star}\right) \text {, and } B_{f}\left(T_{n}^{\star}\right)=\left|\mathbb{E}_{f_{1, n}}\left[T_{n}^{\star}\right]-\left\|f_{1, n}-f_{0}\right\|^{2}\right| .
$$

The, using simple algebra, we get

$$
\begin{aligned}
P_{f_{1, n}}\left(\Delta_{n}^{\star}=1\right) & =P_{f_{1, n}}\left(\left|T_{n}^{\star}\right|>C^{\star} t_{n}^{2}\right) \\
& \leq P_{f_{1, n}}\left(\left|T_{n}^{\star}-\mathbb{E}_{f_{1, n}}\left(T_{n}^{\star}\right)\right|+\left\|f_{1, n}-f_{0}\right\|^{2}+B\left(T_{n}^{\star}\right)>C^{\star} t_{n}^{2}\right), \\
& \leq P_{f_{1, n}}\left(\left|T_{n}^{\star}-\mathbb{E}_{f_{1, n}}\left(T_{n}^{\star}\right)\right|>C^{\star} t_{n}^{2}-\left\|f_{1, n}-f_{0}\right\|^{2}-B\left(T_{n}^{\star}\right)\right) \\
& \leq P_{f_{1, n}}\left(\frac{\left|T_{n}^{\star}-\mathbb{E}_{f_{1, n}}\left(T_{n}^{\star}\right)\right|}{\sqrt{V_{f}\left(T_{n}^{\star}\right)}}>\frac{C^{\star} t_{n}^{2}-\left\|f_{1, n}-f_{0}\right\|^{2}-B\left(T_{n}^{\star}\right)}{\sqrt{V_{f_{1, n}}\left(T_{n}^{\star}\right)}}\right) \\
& \leq\left[\frac{\sqrt{V_{f_{1, n}}\left(T_{n}^{\star}\right)}}{C^{\star} t_{n}^{2}-\left\|f_{1, n}-f_{0}\right\|^{2}-B\left(T_{n}^{\star}\right)}\right]^{2} .
\end{aligned}
$$

In [Butucea 2007], it is proved that $B_{f_{1, n}}\left(T_{n}^{\star}\right) \leq L h_{n}^{2 s}(1+o(1))$ as $n \rightarrow+\infty$. Hence

$$
C^{\star} t_{n}^{2}-\left\|f-f_{0}\right\|^{2}-B\left(T_{n}^{\star}\right) \geq\left(C^{\star}-L\right) t_{n}^{2}(1+o(1)), \text { as } n \rightarrow+\infty .
$$


Since $\sqrt{V_{f}\left(T_{n}^{\star}\right)} \leq O\left(t_{n}^{2}\right)$ as $n \rightarrow+\infty$, we obtain that

$$
P_{f_{1, n}}\left(\Delta_{n}^{\star}=1\right) \leq\left[\frac{C}{\left(C^{\star}-L\right)(1+o(1))}\right]^{2}, \text { as } n \rightarrow+\infty .
$$

Provided $C^{\star}=C_{\xi}^{\star}$ is large enough, we obtain that

$$
\lim _{n \rightarrow+\infty} \sup P_{f_{1, n}}\left(\Delta_{n}^{\star}=1\right) \leq 1-\xi \Rightarrow \lim _{n \rightarrow+\infty} \inf P_{f_{1, n}}\left(\Delta_{n}^{\star}=0\right) \geq \xi .
$$

This result holds whatever the value of $\mathcal{C}_{1}$ in the construction of $r_{n}$. Hence Equation (15) is proved which entails that the test $\Delta_{n}^{\star}$ is not minimax for $H_{0}^{D P}$ on $W\left(s+t, L^{\prime}\right)$.

2nd case: The function $f$ belongs to $S(\alpha, r, L)$ and the noise is polynomially smooth. In this case, following [Butucea 2007, the test $\Delta_{n}^{\star}$ is minimax provided $t_{n}$ and $h_{n}$ are chosen as follows

$$
t_{n}=\frac{1}{\sqrt{n}}\left(\frac{\log (n)}{2 \alpha}\right)^{(\beta+1 / 4) / r} \text { and } h=\left(\frac{\log (n)}{2 \alpha}-\frac{2 \beta+1 / 2}{2 \alpha r_{n}} \log \log n\right)^{-1 / r},
$$

for $\mathcal{C}^{\star}$ large enough. Once again, we consider the function $f_{1, n}$ defined in (13) with

$$
r_{n}=\frac{\mathcal{C}_{1}}{\sqrt{n}}\left(\frac{\log (n)}{2 \alpha}\right)^{1 / 4 r} \geq C \rho_{n}^{D P},
$$

for some fixed constant $C$. This entails

$$
\left\|g *\left(f_{1, n}-f_{0}\right)\right\|^{2}>c_{g} \frac{\mathcal{C}_{1}}{n}\left(\frac{\log (n)}{2 \alpha}\right)^{1 / 2 r}, \text { and }\left\|f_{1, n}-f_{0}\right\|^{2}=\mathcal{C}_{1} r_{n}^{2}=\frac{1}{n}\left(\frac{\log (n)}{2 \alpha}\right)^{1 / 2 r} .
$$

We prove that the test $\Delta_{n}^{\star}$ is not minimax in this situation. Using a similar algebra compared to the first case, we obtain

$$
P_{f_{1, n}}\left(\Delta_{n}^{\star}=1\right) \leq\left[\frac{\sqrt{V_{f_{1, n}}\left(T_{n}^{\star}\right)}}{C^{\star} t_{n}^{2}-\left\|f_{1, n}-f_{0}\right\|^{2}-B\left(T_{n}^{\star}\right)}\right]^{2} .
$$

Using the upper bound obtained in [Butucea 2007, we get

$$
V_{f_{1, n}}\left(T_{n}^{\star}\right) \leq C_{1}\left\|f_{1, n}-f_{0}\right\|_{2}^{2}\left(\log \frac{2 \alpha}{\left\|f-f_{0}\right\|_{2}^{2}}\right)^{2 \beta / r} .
$$

Hence

$$
\begin{aligned}
P_{f_{1, n}}\left(\Delta_{n}^{\star}=1\right) & \leq\left[\frac{\left\|f_{1, n}-f_{0}\right\|_{2}^{2}}{C^{\star} t_{n}^{2}(1+o(1))}\left(\log \frac{2 \alpha}{\left\|f_{1, n}-f_{0}\right\|_{2}^{2}}\right)^{2 \beta / r}\right]^{2}, \\
& =\frac{C_{1}}{C^{\star}} \frac{1}{\log (n)^{2 \beta / r}}\left(\log \left(n C_{1}^{2}\right)\right)^{2 \beta / r} \leq \frac{C_{1}}{C^{\star}}
\end{aligned}
$$


which yields

$$
\lim _{n \rightarrow+\infty} P_{f_{1, n}}\left(\Delta_{n}=1\right) \leq 1-\xi
$$

whatever the value of $\mathcal{C}_{1}$, provided $\mathcal{C}^{\star}$ is large enough.

3rd case: The noise is exponentially smooth and the function $f$ belongs to $W(s, L)$. In this case, the test $\Delta_{n}^{\star}$ is known to be minimax provided

$$
t_{n}=\sqrt{L}\left(\frac{\log (n)}{2 \gamma}\right)^{-s / \beta}, \text { and } h_{n}=\left(\frac{\log (n)}{2 \gamma}-\frac{2 s+1}{2 \gamma \beta} \log \frac{\log n}{2 \gamma}\right)^{-1 / \beta} .
$$

We consider the function $f_{1, n}$ defined in (13) with

$$
r_{n}=\frac{1}{\sqrt{n}}\left(\frac{\log (n)}{\gamma}\right)^{1 / 4 \beta}
$$

This entails

$$
\left\|g * f_{1, n}-g * f_{0}\right\|^{2}>\frac{1}{n}\left(\frac{\log (n)}{\gamma}\right)^{1 / 2 \beta}, \text { and }\left\|f_{1, n}-f_{0}\right\|^{2}=\mathcal{C}_{1} r_{n}=\frac{1}{n}\left(\frac{\log (n)}{\gamma}\right)^{1 / 2 \beta} .
$$

Then

$$
\begin{aligned}
P_{f_{1, n}}\left(\Delta_{n}^{\star}=1\right) & \leq\left[\frac{\sqrt{V_{f}\left(T_{n}^{\star}\right)}}{C^{\star} t_{n}^{2}-\left\|f-f_{0}\right\|^{2}-B\left(T_{n}^{\star}\right)}\right]^{2}, \\
& \leq\left[\frac{h_{n}^{2 s+\beta / 2}}{\left(C^{\star}-1\right) t_{n}^{2}(1+o(1))}\right]^{2}, \\
& \leq \frac{C \log (n)^{-2 s / \beta-1 / 2}}{C^{\star} \log (n)^{-2 s / \beta}} \leq \frac{C}{C^{\star} \log ^{1 / 2}(n)} .
\end{aligned}
$$

Once again, the limit of the above term is bounded from above by $1-\xi$ for a large enough $C^{\star}$.

4th case: The noise is exponentially smooth and the function $f$ belongs to $S(\alpha, r, L)$. For the sake of brevity, we only consider the case where $r<\beta$. In this case, the test $\Delta_{n}^{\star}$ is known to be minimax provided

$$
t_{n}=h_{n}=\exp \left(-\frac{\alpha}{h_{\star}^{r}}\right) .
$$


Remark that $f_{1, n} * g \in S\left(\gamma, \beta, L^{\prime}\right)$. Moreover, choosing

$$
\tau_{n}=\frac{\log (n)^{1 / 4 \beta}}{\sqrt{n}}
$$

we get

$$
\left\|f_{1, n}-f_{0}\right\|^{2}=\tau_{n}^{2}=\frac{1}{n} \log (n)^{1 / 2 \beta} \geq C\left(\rho_{n}^{D P}\right)^{2},
$$

for some positive constant $C$. Then recalling that

$$
\frac{\alpha}{h_{\star}^{r}}+\frac{\gamma}{h_{\star}^{\beta}}=\frac{1}{2}\left[\log (n)-(\log \log n)^{2}\right],
$$

we get that

$$
\left.t_{n}=\exp \left(-\frac{\alpha}{h_{\star}^{r}}\right)=\exp \left(-\frac{1}{2} \log (n)+\frac{(\log \log n)^{2}}{2}+\frac{\gamma}{h_{\star}^{r}}\right) \geq \frac{C}{\sqrt{n}} \exp (\log \log n)^{2}\right) .
$$

Moreover, we get from [Butucea 2007] that

$$
V_{f}\left(T_{n}^{\star}\right) \leq \frac{h_{\star}^{\beta-1}}{n} \exp \left(\frac{2 \gamma}{h_{\star}^{\beta}}-\frac{2 \alpha}{h_{\star}^{r}}\right)+\frac{h_{\star}^{\beta-1}}{n^{2}} \exp \left(\frac{4 \gamma}{h_{\star}^{s}}\right) .
$$

Hence

$$
\begin{aligned}
\frac{\sqrt{V_{f}\left(T_{n}^{\star}\right)}}{C^{\star} t_{n}^{2}} & =\frac{h_{\star}^{(\beta-1) / 2} / \sqrt{n} \exp \left(\gamma / h_{\star}^{\beta}-\alpha / h_{\star}^{r}\right)}{\exp \left(-2 \alpha / h_{\star}^{r}\right)}, \\
& =\frac{h_{\star}^{(\beta-1) / 2}}{\sqrt{n}} \exp \left(\frac{\alpha}{h_{\star}^{r}}+\frac{\gamma}{h_{\star}^{\beta}}\right), \\
& =\frac{h_{\star}^{(\beta-1) / 2}}{\sqrt{n}} \exp \left(\log (\sqrt{n})-(\log \log n)^{2}\right), \\
& =\frac{h_{\star}^{(\beta-1) / 2}}{n} \exp \left(-(\log \log n)^{2}\right) .
\end{aligned}
$$

We can conclude the proof remarking that $h_{\star}^{\beta}$ is of order $(\log (n))^{-1}$ provided $\beta>r$. Hence, the previous term tends to 0 as $n \rightarrow+\infty$.

Acknowledgments. The authors would like to thank the associate editor and two referees for all their constructive remarks that helped to improve the paper. 


\section{References}

[Baraud 2002 ] Baraud, Y. (2002) Non-asymptotic minimax rates of testing in signal detection. Bernoulli, 8, 577-606.

[Baraud et al. 2003 ] Baraud, Y., Huet, S. and Laurent, B. (2003) Adaptive tests of linear hypotheses by model selection, Ann. Statist., 31, no. 1, 225-251.

[Bissantz et al. 2009] Bissantz, N., Claeskens, G., Holzmann, H. and Munk, A. (2009) Testing for lack of fit in inverse regression - with applications to biophotonic imaging. J. Royal Statist. Soc. Ser. B, 71(1), 25-48.

[Bissantz et al. 2007] Bissantz, N., Hohage, T., Munk, A. and Ryumgaart, F. (2007) Convergence rates of general regularization methods for statistical inverse problems and applications. SIAM J. Numerical Analysis, 45, 2610-2636.

[Butucea 2007] Butucea, C. (2007) Goodness-of-fit testing and quadratic functional estimation from indirect observations, Ann. Statist., 35, no. 5, 1907-1930.

[Butucea et al. 2009] Butucea, C., Matias, C. and Pouet, C. (2009) Adaptive goodnessof-fit testing from indirect observations. Ann. Inst. Henri Poincaré Probab. Stat. 45, no. $2,352-372$.

[Butucea et al. 2007] Butucea, C. and Tsybakov, A. B. (2007) Sharp optimality in density deconvolution with dominating bias. I. Teor. Veroyatn. Primen. 52 , no. 1, 111-128; translation in Theory Probab. Appl. 52 (2008), no. 1, 24-39

[Cavalier 2008] Cavalier, L. (2008) Nonparametric statistical inverse problems, Inverse Problems, 24(3), 1-19.

[Delaigle et al. 2004] Delaigle, A. and Gijbels, I. (2004) Bootstrap bandwidth selection in kernel density estimation from a contaminated sample. Ann. Inst. Statist. 56, 19-47.

[Engl et al. 1996] Engl, H.W., Hanke, M. and Neubauer, A. (1996) Regularization of Inverse Problems. Kluwer Academic Publishers Group, Dordrecht.

[Fan 1991] Fan, J., (1991) On the optimal rates of convergence for nonparametric deconvolution problems. Annals of Statistics, 19, 1257-1272.

[Holzmann et al. 2007] Holzmann, H., Bissantz, N. and Munk, A. (2007) Density testing in a contaminated sample. Journal of Multivariate Analysis, 98, 55-75.

[Ingster 1993] Ingster, Yu. I. (1993) Asymptotically minimax hypothesis testing for nonparametric alternatives I-II-III. Math. Methods Statist., 2, 85-114, 171-189, 249-268. 
[Ingster et al. 2012] Ingster, Yu.I., Sapatinas, T. and Suslina, I.A. (2012) Minimax signal detection in ill-posed inverse problems. Annals of Statistics, 40, 1524-1549.

[Laurent et al. 2012] Laurent, B., Loubes, J-M. and Marteau, C. (2012) Non asymptotic minimax rates of testing in signal detection with heterogeneous variances. Electronic Journal of Statistics. Volume 6, pp 91-122.

[Laurent et al. 2011] Laurent, B., Loubes, J-M. and Marteau, C. (2011) Testing inverse problems: a direct or an indirect problem? Journal of Statistical Planning and Inference, 141 , pp. 1849-1861.

[Loubes et al. 2009] Loubes, J-M. and Rivoirard, V. (2009) Review of rates of convergence and regularity conditions for inverse problems. Int. J. Tomogr. Stat., (11) S09, 61-82.

[Meister 2009] Meister, A. (2009) Deconvolution in nonparametric statistics. Lecture notes in statistics, Springer. 